\title{
Strong Coupling between ZnO Exciton and Localized Surface Plasmon in Ag Nanoparticles Studied by STEM-EELS
}

\author{
Jiake $\mathrm{Wei}^{1,2}, \mathrm{Jia} \mathrm{Xu}^{3}$, Xuedong $\mathrm{Bai}^{2}, \mathrm{Jingyue} \mathrm{Liu}^{1}$
}

1. Department of Physics, Arizona State University, Tempe, Arizona 85287, USA

2. Beijing National Laboratory for Condensed Matter Physics, Institute of Physics, Chinese Academy of Sciences, Beijing, 100190, China

3. School for Engineering of Matter, Transport and Energy, Arizona State University, Tempe, Arizona 85287, USA

Metallic nanoparticles (NPs) in combination with semiconductor nanostructures have been intensively investigated recently. The complementary optical properties of two composite units, with long-live excitonic excitations in semiconductor nanostructures and localized electromagnetic modes in metal NPs, provide the possibility to modify and design specific optical responses and to observe new phenomena based on exciton-plasmon coupling [1]. For example, recent studies show enhanced emission, luminescence emission wavelength shift and the nonlinear Fano effect in the semiconductor-metal nanostructures [2]. We report here scanning transmission electron microscopy-electron energy loss spectroscopy (STEM-EELS) study on the coherent coupling between excitons in ZnO nanowires (NWs) and localized surface plasmons (LSPs) in Ag NPs.

The Ag/ZnO NWs were synthesized by a modified evaporation deposition method. The EELS experiments were conducted on a Nion UltraSTEM ${ }^{\mathrm{TM}} 100$ equipped with a monochromator, a C3/C5 aberration corrector, and a Gatan Enfina electron energy-loss spectrometer. The monochromated STEMEELS system can achieve an energy resolution $<15 \mathrm{meV}$ as well as a spatial resolution $<0.1 \mathrm{~nm}$ at 60 $\mathrm{KV}[3]$.

Figure 1a shows the high-angle annular dark-field (HAADF) image of Ag nanoparticles supported on a $\mathrm{ZnO}$ NW. Figure 1b shows the EELS spectrum of a pure ZnO NW (black curve) and an individual Ag particle with a diameter of $\sim 15 \mathrm{~nm}$ (shown in the inset), supported on a $10 \mathrm{~nm}$ thick $\mathrm{Si}_{3} \mathrm{~N}_{4}$ substrate (red curve). The excitonic peak of pure $\mathrm{ZnO} \mathrm{NW}$, located at approximately $3.42 \mathrm{eV}$, is very weak due to the phonon broadening effect. The LSP resonance energy of the Ag particle is $3.29 \mathrm{eV}$, attributable to the dipole mode. For the $\mathrm{Ag} / \mathrm{ZnO}$ composite system, coupling of the $\mathrm{ZnO}$ excitons with the Ag LSPs leads to the formation of two new exciton-plasmon polaritons (upper polariton (UP) and lower polartiton (LP), shown in Fig. 1c). When the electron beam was positioned on the edge of the NP (position 1 in the inset), both UP and LP were excited. However, when the electron beam was moved to position 2, only UP was highly excited. The peak at $~ 2.75$ arises from the interface plasmon of $\mathrm{Ag}$ and $\mathrm{ZnO}$. Figure 2 shows a series of EELS spectrum for different sizes of Ag NPs varying from $4.4 \mathrm{~nm}$ to $42.5 \mathrm{~nm}$. Figure $2 \mathrm{a}(2 \mathrm{~b})$ was acquired when the beam was put on location 1(2) in the inset of Fig. 1c. For pure individual Ag NPs, a significant blueshift of the LSP resonance energy from $3.2 \mathrm{eV}$ to $3.7 \mathrm{eV}$ has been reported when the size of the Ag NPs decreases from about $30 \mathrm{~nm}$ to 2-3 nm [4]. In our Ag/ZnO system, the EELS spectra show a clear anti-crossing behavior of the UP (dotted red arrow in Fig. 2a) and LP (dotted red arrow in Fig. $2 b)$ at the energy of the $\mathrm{ZnO}$ exciton $(\sim 3.42 \mathrm{eV})$, which indicated by the dotted black line in Fig. $2 \mathrm{a}$ and $2 \mathrm{~b}$. The interface plasmon peaks $(2.60-2.75 \mathrm{eV})$ in Fig. $2 \mathrm{~b}$ are sensitive to the specific structure of the interfacial region. This work demonstrates that the monochromated STEM-EELS is a 
powerful tool for studying the plasmon-exciton coupling in metal/semiconductor composite systems on a nanometer scale [5].

\section{References:}

[1] M. Achermann, J. Phys. Chem. Lett. 1 (2010), p.2837.

[2] W. Zhang, A. O. Govorov and G.W. Bryant, Phys. Rev. Lett. 97 (2006), p. 146804.

[3] O. L. Krivanek et al., Nature, 514 (2014), p. 209.

[4] J. A. Scholl, A. L. Koh and J. A. Dionne, Nature 483 (2012), p. 421.

[5] The authors acknowledge the College of Liberal Arts and Sciences of Arizona State University for funding and the use of the John M. Cowley CHREM facilities at Arizona State University.


Figure 1. (a) HAADF image of the $\mathrm{Ag} / \mathrm{ZnO}$. (b) The EELS spectrum of a pure $\mathrm{ZnO} \mathrm{NW}$ black) and $\mathrm{Ag}$ nanoparticle (red) with a diameter of $15 \mathrm{~nm}$ (inset). (c) The EELS spectra of the Ag/ZnO NW (inset) from different positions. The size of the Ag particle is similar to the one in (b). All spectra were normalized by the zero loss peak intensity.


Figure 2. (a) A series of EELS spectrum obtained with different sizes of Ag NPs when the electron beam was located at position 1 in Fig. 1c. (b) EELS spectra obtained from the position 2 in Fig. 1c. The dotted black line indicates the energy of the $\mathrm{ZnO}$ exciton. The dotted red arrow indicates the blueshift of the LP and UP energy. 